\title{
Apport du sport dans la lutte contre l'insécurité à l'aune de recrudescence des staffs dans la ville de Lubumbashi
}

\author{
Par MUSONDA KISHIMBA Jean-Marie*
}

\section{INTRODUCTION}

La ville de Lubumbashi traverse une criminalité dont l'une des causes ne saurait mettre en marge l'engouement provoqué par les activités communément appelées "Staffs » qui deviennent de plus en plus des structures urbaines imposantes. Autant que se multiplient les églises, les Staffs gagnent de plus en plus du terrain, chaque Commune ou quartier de la ville en compte un nombre considérable. La sous-estimation d'un encadrement propice de ce phénomène nouveau n'a pas épargné le présent article d'analyser les effets pervers, le mutisme et l'inaction des pouvoirs publics dans la prévention ainsi que dans la prise des précautions pour réguler l'impact et l'ascendance du phénomène staff.

Par ailleurs, la question permettra sans doute d'analyser en même temps l'apport du hard power reconnu aux pouvoirs publics dans la gestion des effets pervers des staffs qui touchent à l'ordre public urbain. En effet, l'influence négative de l'insécurité sur le fonctionnement normal des institutions et le développement des quartiers devenant une préoccupation de tous il s'impose l'implication globale des facteurs du soft power devant contribuer au maintien de l'ordre public. A partir de ces perspectives, l'intégration du sport dans la lutte contre l'insécurité devient un moyen de repenser les efforts de lutte et tire son origine dans la considération de sport en général comme une activité qui occupe une place de choix dans la vie nationale de par ses fonctions éducatives, culturelles, sanitaires, politiques, sociales et économiques. En tant qu'instrument de puissance économique et organisationnelle des Etats, le pouvoir symbolique du sport nécessite de jouer le véritable rôle du sapeur-pompier contre l'insécurité grandissante dans la ville de Lubumbashi. A cet effet, le recours au sport s'inscrit dans ses atouts réels à contribuer comme moyen de prévention et de précaution en tant que vecteur d'occupation et de développement des jeunes.

Par ailleurs, la sécurité et la tranquillité étant des éléments moteurs de l'ordre public, ils demeurent le fondement du développement économique et social « en tant qu'ensemble des valeurs essentielles à la société considérée à une époque bien déterminée, dont la sauvegarde et le respect s'imposent à tous y compris le pouvoir public $»^{1}$. A cet effet, la législation congolaise et la doctrine ne s'empêchent pas d'estimer que « les activités physiques et sportives occupent une place pouvant créer et restaurer le lien social, canaliser la violence

* Chef de travaux à la Faculté de Droit de l'Université de Lubumbashi/Département de droit économique et social.

1 Joseph TSHIBASU PANDAMADI, «La problématique de la répression du trafic illicite des stupéfiants en RDC et son incidence dans l'ordre public national et international », in Revue Justitia Faculté de Droit/Université de Lubumbashi, 2018, p.85. 
et favoriser la citoyenneté. $»^{2}$. Dans le même ordre d'idée, Alain Ferrand réaffirme que « l'univers sportif est un formidable levier de développement, d'intégration et de progrès social $»^{3}$. S'inscrivant dans la vision d'impulser une nouvelle dynamique du sport dans le développement, Emmanuel Tessier souligne que « La victoire en matière de stades doit être à la fois politique, économique, architecturale et sportive $»^{4}$. Pour sa part, Pamela Echevirria réaffirme que « l'insécurité urbaine est un phénomène mondial $»^{5}$ et fait de plus en plus l'objet de réflexion et d'analyse sur les opérations des staffs en tant que structures urbaines négatives.

Pour ce faire, la valorisation du rôle du sport dans la régulation d'implantation et des méfaits de staffs dans la ville de Lubumbashi dont l'appréhension et la pratique outrepassent le sens normal « de l'équipe dirigeante d'une entreprise » à l'instar du club ou autre, en devenant des lieux de trafic où se rencontrent l'offre et la demande des liqueurs fortes, des stupéfiants communément appelées « zododo » et « kabondo » ${ }^{6}$, des chanvres et drogues, des lieux de concentrations de jeunes et des structures asiles de prostitution, des foyers des barbaries et des centres de négoce des biens d'occasion ou de secondes mains, oriente l'ensemble composite d' actions en vue d'étouffer les stratégies désastreuses des staffs. Il s'avère, dès lors, utile d'inscrire notre réflexion dans une démarche exploratoire.

$\mathrm{Au}$ vu de ce qui précède, la multiplication desdits mouvements n'étant pas neutres à l'insécurité dans la ville cuprifère, ils influencent également le système sécuritaire dans la mesure où de ce phénomène émergent les facteurs qui alimentent la montée en puissance du commerce clandestin et illégal des stupéfiants, l'espionnage ainsi que la justice populaire. Dans une approche de la gestion de l'ordre public dans la Province du Katanga, le professeur Jean-Marie Dikanga Kazadi a pu désigner parmi les grands défis inscrits au registre des éléments perturbateurs de l'ordre public les manifestations sportives de football ${ }^{7}$. Au regard du contexte actuel de l'ordre public, les manifestations sportives de football constituent la partie visible de l'iceberg ou encore l'arbre qui cache la forêt dans la mesure où

2 Rapport présenté par André LECLERCQ, le sport au service de la vie sociale, 2007, p.I.28. Lire aussi l'Exposé des motifs de la Loi n ${ }^{\circ} 11 / 023$ du 24 décembre 2011 portant principes fondamentaux relatifs à la l'organisation et à la promotion des activités physiques et sportives en RDC.

3 Alain FERRAND, "Mécénat » in dictionnaire culturel du sport, éd. Armand Colin, Paris, 2010, p.342.

4 Emmanuel TESSIER, Le stade en droit public recherche sur le régime juridique des enceintes sportives, éd.L'Harmattan, Paris, 2015, p.555.

5 Pamela ECHEVIRRIA, "L'impact de l'insécurité urbaine sur les entreprises manufacturières d'Amérique latine : une étude de cas Puebla au Mexique » thèse soutenue en 2009, p.3.

6 «Zododo »désigne dans le langage lushois des liqueurs fortes et « Kabondo » est l'alcool produit localement est appelé vendus dans des bouteilles de 5 à 10 centilitres et en poudre à moindre coût et très enivrant.

7 Jean-Marie DIKANGA KAZADI, «La gestion de l'ordre public dans la province du Katanga » in frontières et gouvernance sécuritaire pour le développement de la République Démocratique du Congo (dir Jean-Marie DIKANGA KAZADI et Emmanuel BANYWESIZE), éd. L'Harmattan, Paris, 2013, p.21. 
derrière les éléments perturbateurs de nature sportive s'imbriquent la multiplicité des facteurs endogènes et exogènes. Ces derniers privilégient, ainsi, l'étude du phénomène récent « les staffs » à partir de ses effets pervers. Par la même occasion, le constat se justifie à travers le lien de causalité universellement admis entre les stupéfiants et la perturbation de santé physique et morale dans tous les secteurs de la vie humaine provoquant ainsi le changement de comportement y compris dans le domaine du football.

Quoique ce phénomène nouveau soit complexe et de nature controverse à analyser sous l'angle de droit, les préoccupations demeurent tout de même sur le pourquoi de l'évolution et de la prolifération grandissante en toute quiétude du phénomène «staffs »; le comment fonctionnent-ils, les actions synergiques pour limiter leurs conséquences de nuisance à l'ordre public et enfin la place ou l'implication des pouvoirs symboliques du sport à servir des vecteurs de transformation des muscles en proie à l'abandon et à la barbarie en des muscles qui coïncident avec de nombreuses aspirations à la pointe du combat d'instauration de la paix et du développement durable.

Il s'agit là d'une contribution à la lutte contre l'insécurité et l'instauration d'un Etat de droit dont la nécessité qui conduit aux efforts synergiques, s'avère l'apanage de toute la population lushoise (habitants de la ville de Lubumbashi). A l'heure actuelle, les qualités intrinsèques de de hard power et de soft power sur les problématiques sociales, économiques, culturelles ou encore environnementales en vue d'améliorer la situation sécuritaire procurent une occasion de concilier leur interférence. Pour y parvenir, la présente réflexion préconise l'analyse du phénomène " staffs » dans ses aspects fonctionnaliste et structural qui servira de moyen pour appréhender l'origine et la recrudescence des actes de vandalisme, de vol et de viols enregistrés ces derniers temps dans la ville de Lubumbashi. En effet, il s'avère indéniable de noter que les conséquences néfastes de consommation des stupéfiants sur la santé physique et morale de l'humanité confortant les effets de trouble de comportement à la base de criminalité, un regard s'impose sur l'analyse fonctionnelle et sur les enjeux des staffs en tant qu'un des éléments matériels de l'insécurité.

\section{A. LES STAFFS COMME RECEPTACLE MATERIEL ET FONCTIONNEL DE L'INSECURITE.}

L'avènement des staffs comme nouveau phénomène à impact visible et négatif dans la ville nécessite de circonscrire les aspects constitutifs et les caractéristiquesliées à ce phénomène dans les points qui suivent.

\section{LES STAFFS EN TANT QU'OBJET DE CONCENTRATION DE JEUNES}

La permanence de concentration de jeunes autour de structures connues dans les quartiers de la Ville de Lubumbashi dénommées « staffs» occupe une place importance dans l'instauration d'un Etat de droit et dans la lutte contre l'insécurité dont la gravité n'échappe aux multiples réflexions à de niveaux différents pour combattre ce fléau. A cet effet, tous les 
problèmes relatifs à l'insécurité que pose la participation accrue de la jeunesse implique la mobilisation pluridisciplinaire dont les sciences juridiques dans la question de la gestion de la chose publique au regard de l'assainissement du milieu.

\section{Définition du staff}

Il convient d'estimer que le staff n'est pas un concept qui est défini en droit congolais si bien que les conséquences qui en découlent ne sont pas en marge du droit et des débats interdisciplinaires. L'impasse de la définition fiable permet de noter en considérant le point de vue pratique qu'un staff constitue un lieu où se rassemblent les jeunes, où se commercialisent de manière illicite des stupéfiants et où les jeunes qui fréquentent le milieu se font appeler membres en lieu et place des clients.

De prime abord, il sied de penser qu'un staff serait par nature un marché informel ou encore un lieu d'exploitation commerciale où se rencontre l'offre et la demande en toute clandestinité. Par ailleurs, l'univers fonctionnel et matériel des staffs échappent considérablement à la simple qualification du marché informel sur plusieurs observations concernant : leur soutien, les catégories des clients et leurs âges, leurs orientations idéologiques, les sources d'approvisionnement, etc.

S'agissant de leur soutien, le terrain dans lequel opère un staff nous renseigne que plusieurs personnes apportent leurs contributions au progrès des staffs parmi lesquelles : les clients appelés « membres » dont l'âge varie entre treize à vingt-cinq ans qui s'abreuvent en produits enivrants et dont les occupations professionnelles sont variées. Certains œuvrent comme vendeurs des carburants sur les voies publiques et d'autres sont soit receveurs dans des taxis bus ou chauffeurs soit encore des mécaniciens tandis que la majorité se livre aux jeux de cartes ou de dames payant.

Concernant leurs orientations idéologiques, certaines formations politiques trouvent dans les concentrations des jeunes des potentiels militants très zélés pour asseoir toutes les revendications politiciennes et formations d'identité d'appartenance à leur leadership.

Par ailleurs, certains staffs se revendiquent à tort ou à raison d'être soutenus et reconnus par les autorités politico-administratives et militaires comme des groupes de renseignement sur les évènements des quartiers, de formation idéologique voire de souches de règlement de compte dès lors qu'une plainte est formulée moyennant un gain, il s'en suit la justice populaire ou le règlement de compte.

En plus, certains des membres au sens pratique des staffs sont aussi recrutés par des églises en devenant des adeptes des chorales communément dénommées " canon vocal ».

De tout ce qui précède, les aspects définitionnels illustrent indéniablement la difficulté de déterminer clairement la nature juridique. A tout le moins, la présente réflexion ne peut que tenter une qualification littérale des staffs comme des mouvements socio-économiques d'utilité politique, religieuse, sexuelle et de justice populaire dont le principal effet ne peut que la pluralité d'actions. 
2. Encadrement des staffs et développement de nouvelles formes des violences

$\mathrm{Au}$ regard de leur fonctionnement, chaque staff est chapoté par un responsable qui est le détenteur d'un local ou d'une maison et qui assure l'orientation et coordonne les activités du mouvement. Analysant l'incidence entre la prolifération des staffs et la vente illicite des drogues, chanvres et le zododo, il convient de noter avec le professeur Joseph Tshibasu que « l'usage de la drogue constitue un facteur de trouble à l'ordre public du fait de l'impact de l'abus de la drogue et l'addiction qu'il entraine sur la criminalité et la violence. Il existe un lien tangible entre criminalité, violence et ingestion de drogue en ce sens que certains toxicomanes recourent à la violence pour financer leur accoutumance, ce qui les oblige à se livrer à des vols et des séries d'agression sur leur entourage $»^{8}$.

Dès lors qu'il est établi que les stupéfiants constituent les intrants à la criminalité, il n'est pas, par ailleurs, exclu que les différents endroits d'approvisionnement puissent être classés parmi les causes d'insécurité qui accable la ville cuprifère dans la mesure où les effets pervers de la consommation des stupéfiants entrainent la perturbation de comportement des individus. Il s'en suit que la pointe du combat contre ce phénomène puisse s'orienter sur les individus, les points des distributions ainsi que sur leurs différentes sources d'approvisionnement dès lors que sur le plan du droit la lutte contre le trafic illicite des stupéfiants s'estime comme une action universelle qui s'impose à tous.

\section{a) Du point de vue national}

Le contour de la répression a pour fondement le décret du $1^{\text {er }}$ mars 1903 portant sur les mesures contre l'usage de fumer le chanvre. En plus, la loi º11/023 du 24 décembre 2011 portant principes fondamentaux relatifs et à l'organisation et à la promotion des activités physiques et sportives en République Démocratique du Congo place l'importance à la lutte et au contrôle du dopage dans le sport en interdisant dans ses articles 74 et 75 « la production, la circulation, l'importation, la distribution et la vente des substances à impact de performance artificielle et la commercialisation et la distribution des compléments alimentaires $»^{9}$.

b) Sur le plan international

Au niveau international, plusieurs instruments juridiques coordonnés de lutte contre les stupéfiants existent à l'instar de la convention unique sur les stupéfiants de 1961 telle modifiée

8 Joseph TSHIBASU PANDAMADI, op.cit, p.95. On peut apprendre de l'auteur que « le trafic illicite de la drogue constitue des sources de financement du terrorisme et l'argent généré par ce crime est souvent soumis à un blanchiment des capitaux et permet de soutenir ainsi d'une manière permanente ce crime organisé ».

9 Articles 74 et 75 de la loi n ${ }^{\circ} 11 / 023$ du 24 décembre 2011 portant principes fondamentaux relatifs et l'organisation et à la promotion des activités physiques et sportives en République Démocratique du Congo. 
par le protocole de 1972, la convention sur les substances psychotropes ainsi que la convention des nations unies contre le trafic illicite de stupéfiants et de substances psychotropes de $1988^{10}$. Tous ces instruments juridiques justifient le recours à la force (hard power) et aux mécanismes dissuasifs comme stratégies pour les pouvoirs publics empêchant les conséquences néfastes.

\section{LE HARD POWER DANS L'INSTAURATION DE LA SECURITE}

1. Le maintien de l'ordre public

Il convient de noter que le hard power est un moyen légitime dans le maintien de l'ordre public et vise au niveau interne pour chaque Etat de veiller à la sécurité publique, à la tranquillité publique, à la salubrité publique et à la moralité. Ces éléments constitutifs de l'ordre public fondent l'Etat à recourir à la Police nationale qui impose une force dissuasive en tant qu'organe habilité pour assurer l'ordre public. Par ailleurs, la gestion de l'ordre public pour un phénomène récent des Staffs dont l'ampleur est actuelle démontre quelques contradictions actantielles de l'organe chargé à assumer cette fonction. Ces contradictions se déclinent dans le contrôle des produits stupéfiants.

\section{a) Au niveau des frontières}

Les dispositifs de contrôle des liqueurs fortes vendues dans des petites bouteilles paraissent d'une observation stricte. Par contre une fois ces produits traversés illégalement ou produits localement échappent à l'interception des organes habilités, ils deviennent immunisés de tout contrôle et toute sommation en devenant commerçables dans tous les grands marchés de la ville. Ainsi, la prolifération des staffs trouvent des sources d'approvisionnement dans leur proximité y compris en boissons très alcooliques de production locale.

b) Les difficultés de gestion de l'ordre public par le hard power

Pour une présentation des difficultés, le professeur Jean-Marie Dikanga Kazadi, a réalisé que « la gestion de l'ordre public dans le Grand Katanga bute sur deux difficultés majeures : la première est d'ordre matériel et logistique et la deuxième est d'ordre humain. Pour la première, l'auteur fait allusion aux moyens conséquents et utiles à mettre à la disposition de ceux qui agissent au nom de l'Etat pour le maintien de l'ordre public. Quant à la deuxième, l'auteur fait allusion aux effectifs en nombre inferieur et aussi l'insuffisance de formation $»^{11}$.

10 / ONUDC, les conventions internationales relatives au contrôle des drogues, édition révisée 2013.

11 Jean-Marie DIKANGA KAZADI, «La gestion de l'ordre public dans la province du Katanga » in frontières et gouvernance sécuritaire pour le développement de la République Démocratique du Congo, Paris, 2013, p.23. 
S'agissant de difficulté relative à la formation, Gauthier Viller et alliés ont affirmé qu'à « l'entrée de l'Alliance des Forces Démocratiques pour la Libération (AFDL), la sécurité des personnes et de leurs biens dans la ville de Lubumbashi après le départ des unités combattantes dans d'autres Provinces non encore libérées, était assurée par les personnes enrôlées de tout bord. En effet des civils sans formation et tous les bandits qui se sont évadés des prisons $»^{12}$. Sur plusieurs situations évoquées, il y a lieu de reconnaitre une évolution dans la mesure où la Police congolaise subie des formations et est régulièrement dotée des matériels. Par contre, les défis sécuritaires face au phénomène staff demandent de cerner la difficulté de la professionnalisation des policiers dans la mesure où l'insécurité dans la ville de Lubumbashi est à situer à deux niveaux. Le premier est celui qui rime avec les actes de vol en pleine journée avec le phénomène enfants de la rue et pick poker dont la plupart se pointent aux lieux où se trouvent la Police de circulation routière et la deuxième plus violente se déroulant la nuit.

\section{c) Difficultés d'action au niveau interne}

Il sied de noter avec Niagalé Bagayoko-Penone qu'au sujet des problèmes d'intervenir avec efficacité sur la recrudescence des violences seraient « à la fois la maitrise de l'information, la maitrise des espaces et la maitrise de violence $»^{13}$. Par contre, la situation géographiquement connue de certains staffs en plein centre-ville (le croisement des avenues du Trente juin, Tabora et Aéroport et Lumumba) dans la Commune Annexe (les avenues de la Mission et Mpangula) pour ne citer qu'à titre d'exemple ces endroits exclut l'ignorance de l'existence de ces structures.

S'agissant de la maitrise de l'information l'appartenance de certains hommes en uniforme au rang des membres ci-haut évoqués exclut toute ignorance sur le phénomène staff.

En ce qui concerne la maitrise des violences, il y a lieu de noter que certains chefs des staffs font l'objet d'interpellation de routine aux différents Commissariats de Police des quartiers mais la promptitude de leur relâchement donne matière à réflexion.

Dans un tel environnement, pallier aux difficultés qui résultent dans l'impact négatif du hard power dans la gestion des effets pervers qui touchent aux questions de l'ordre public à l'aune de ce phénomène nouveau, nécessite la prise en compte du soft power qu'incarne le sport.

12 Gauthier de VILLERS Jean-Claude WILLAME, Jean OMASOMBO et Erik KENNES, République démocratique du congo Chronique politique d'un entre- deux- guerres octobre 1996-juillet 1998, éd. L'Harmattan, Paris, 1998, p.110.

13 Niagalé GAGAYOKO-PENONE, Afrique : les stratégies française et américaine, L'Harmattan, Paris, p. 370 . 


\section{B. LES MECANISMES DE CONVERSION DES MUSCLES D'INSECURITE EN MUSCLES CONTRIBUTEURS AU DEVELOPPEMENT DE LA VILLE}

Les différentes mutations que connait le sport permettent au monde de s'accorder et de reconnaître son énorme potentiel, sa portée mondiale, son langage universel, son impact sur l'économie mondiale, sur les communautés en général et sur les jeunes en particulier ${ }^{14}$. Les staffs n'étant pas en marge des moyens qui contribuent au détournement de la jeunesse, le secteur sportif et l'exploitation du pouvoir du sport au service du développement et de la paix se présente comme un atout majeur d'encadrement et d'impact sur le développement socio-économique en République Démocratique du Congo en prenant le cas du football.

\section{L'INTEGRATION DU SPORT AUX FACTEURS DU DEVELOPPEMENT}

1. La place du sport dans le système de vie urbaine.

Le droit régissant les sports constitue la pièce maitresse qui non seulement facilite la création des structures, mais aussi fixe des conditions susceptibles de rendre le domaine sportif comme un outil efficace d'occupation des jeunes, de développement et de transformation des sports en un facteur de développement durable. En effet, l'intégration des sports aux facteurs du développement tire son origine de son imbrication dans le domaine économique tandis que sa place de choix dans la vie nationale s'affirme de plus en plus à travers les multiples fonctions sus évoquées.

\section{L'incorporation du secteur sportif dans l'environnement juridique congolais}

Partant de la constitution du 18 février 2006 telle que modifiée par la loi $n^{\circ} 11 / 002$ du 20 janvier 2011, la loi $\mathrm{n}^{\circ} 11 / 023$ du 24 décembre 2011 portant principes fondamentaux relatifs à l'organisation et à la promotion des activités physiques et sportives en RDC, les mesures d'application en termes d'arrêtés ministériels voire des circulaires du ministre ayant les sports dans ses attributions, matérialisent le sport comme un service public d'appui au développement et permet audit secteur d'être inscrit au centre des débats de solution pour la tranquillité de la ville. Thierry Lardinoit et Gary Tribou ont souligné, à ce propos « qu'aujourd'hui, le sport est un secteur économique à part entière et mérite d'être l'objet de recherche. En tant que tel, il présente un intérêt pluridisciplinaire manifeste économique, mais aussi philosophique, anthropologique, psychologique, juridique ou politique voire interdisciplinaire $»^{15}$. A cet effet, l'objet d'appréhender les voies et moyens de contribution du do-

14 Position adoptée par le Bureau de l'observateur permanent du Comité International Olympique auprès des Nations Unies sous le thème « La contribution du sport aux objectifs de développement durable et au programme de développement pour l'après 2015 » au nom du mouvement olympique et sportif du février 2015.

15 Thierry LARDINOIT et Gary TRIBOU, «Quelle est la spécificité de la gestion du sport? » in Revue française de Gestion dossier de gestion du sport, vol.30, n¹50, éd. Lavoisier, 2004, p.127. 
maine sportif aux objectifs d'encadrement des jeunes du point de vue socio-économique serait également l'applicabilité de tous les moyens qui s'imposent y compris le Droit. Comme le rappelle justement le professeur Paul Musafiri, « le sens principal du Droit consiste à former, fixer et développer un certain régime de relations sociales dont le but essentiel est de promouvoir entre les membres de la société un certain type d'intercommunication susceptible de garantir la promotion individuelle et collective, culturelle et économique, politique et sociale. La mission primordiale consiste dès lors de trouver solution au problème du développement socio-économique ${ }^{16}$.

$\mathrm{Au}$ vu de ce qui précède, la propension envahissante de l'insécurité étant consolidée par les causes diverses économiques consistant à voler de l'argent ou des biens de valeur et sociales dans le cadre du chômage, la non occupation des jeunes, la pratique des sports dans un environnement sûr et sain devient de plus en plus le moyen par lequel les organes de l'Etat et des institutions sportives privées doivent s'entremêler dans la gestion des activités sportives comme un vecteur de cristallisation de l'ordre public.

3. Le sport en tant que facteur capable de contribuer à l'emploi et aux finances privées.

Comme le démontre Jean-Remi Cognard, « les compétitions sportives sont devenues des spectacles en soi pour lesquels les passionnés sont prêts à payer pour avoir le droit d'y assister ou simplement de les regarder à la télévision ${ }^{17}$. Ce postulat conduit, de manière générale, à la prise de conscience des pouvoirs publics, mais aussi doit permettre de capitaliser l'intérêt relatif aux valeurs fondamentales éducatives, culturelles, économiques, politiques et sociales véhiculées par le monde du sport en général. En prenant en particulier le football en tant qu'une réalité pratique qui se développe de plus en plus, le constat est indubitable que les joueurs qui émergent dans le football ne sont pas tous universitaires. Par ailleurs, les jeunes qui sont en difficulté de scolarité étant traités comme inutiles dans la vie urbaine et trouvent dans des structures des staffs comme de cadres de refuge et dans les violences comme leurs sources financières. Et pourtant, la grille de lecture de l'organisation des formes juridiques du sport démontre qu'en RDC toutes les activités sportives peuvent se pratiquer avec un rôle précis.

4. Formes juridiques des pratiques des activités sportives et leurs rôles

Au regard de la loi sportive sus visée, consacre un chapitre sur les formes des activités physiques et sportives avec leurs fonctions précises.

16 Paul MUSAFIRI, Pièges symboliques et violation des Droits Humaines en Droit positif congolais contribution critique à l'élaboration du Droit praxéologique, éd. La Dialectique, Lubumbashi, 2014, p.20-21.

17 Jean-Remi COGNARD, Contrat de travail dans le sport professionnel, Juris éditions, coll. Horssérie, 2012, p.5. 
a) L'éducation physique et sportive et un sport de masse

L'éducation physique et sportive, EPS en sigle, occupe un aspect pédagogique et s'impose dans le système global de l'enseignement comme un moyen d'asseoir le développement éducatif, culturel et scientifique. Cet aspect rime avec la logique internationale dans la matérialisation de la déclaration de Punta Del Este sur l'importance de l'EPS comme une partie intégrante et essentielle dans le processus du développement humain, sanitaire et social.

Un sport de masse est, à ce titre, celui qui constitue une activité de type récréatif qui vise la détente des personnes qui s'y adonnent. L'importance qu'affiche cette catégorie est de favoriser l'initiation à la pratique, la détection des talents et leur orientation sportive. Sur fondement de l'article 7, la loi sportive intègre une autre dimension universelle des droits de toute personne au repos et aux loisirs.

b) Le sport de compétition et le sport de haut niveau

Le sport de compétition qui se conçoit dans la comparaison des performances entre les personnes physiques ou morales regroupées en clubs ou équipes, organisée par les fédérations nationales ou internationales. A ce propos, l'article 8 de la loi sportive démontre que «le sport de compétition est toute activité physique à caractère de jeu sous forme d'une lutte avec soi-même ou avec autrui dans le cadre d'une structure et des règles établies par des instances internationales. Il permet à tout athlète d'exploiter individuellement ou en équipe ses capacités physiques, morales et psychiques dans la réalisation des meilleures performances sportives ». Au niveau national, le sport de compétition permet de sélectionner les meilleurs talents nationaux pour l'équipe nationale dont l'effort est de représenter la nation aux compétitions internationales.

Le sport de haut niveau, aux termes de la loi sportive, rime avec une activité pratiquée par l'élite visant la réalisation du résultat et la performance évaluée par rapport à des normes techniques internationales. Il requiert un effort constant et un travail en profondeur. Il est développé et soutenu en priorité dans les disciplines sportives olympiques ainsi que dans celles où sont organisées des compétitions de niveau international.

c) Le handisport, le sport-loisir, le sport amateur de masse et le sport professionnel

Le handisport permet d'organiser le sport en vue de l'insertion ou la réinsertion socioprofessionnelle des personnes vivant avec handicap, dans l'objectif d'assurer auxdites personnes toute autonomie physique. La pratique du handisport repose sur le principe du sport en tant qu'un droit fondamental pour tous et comme un moyen de lutte contre la discrimination entre les personnes sur base de leur condition physique. Pour ce faire, l'article 20 alinéa 3 de la loi sportive dispose avec un intérêt particulier que « les structures sportives qui organisent et promeuvent la pratique des activités physiques et sportives à l'intention des personnes vivant avec handicap bénéficient de l'aide des pouvoirs publics, notamment en 
matière d'accès aux équipements sportifs adaptés, d'organisation des compétitions, de formation des encadreurs spécialisés et d'adaptation de transport ».

Le sport-loisir est celui qui a pour objet l'épanouissement physique, moral et social de l'individu ainsi que le développement de ses capacités pour des raisons de santé ou d'insertion sociale. Suivant les prescrits légaux, le caractère dudit sport est essentiellement récréatif.

Le sport amateur de masse est, contrairement aux sports de type récréatif, un sport qui emploie tout pratiquant licencié et affilié par un club qui s'adonne à une discipline sportive par amour du jeu. Cette forme ne permet pas à un sportif d'exiger un profit financier. Cette vision des choses rencontre Norbert Elias et Eric Gunning lorsqu'ils estiment que « la composante principale de l'éthique amateur est l'idée que l'on pratique le sport pour s'amuser $»^{18}$.

Enfin, le sport professionnel est celui pratiqué par un athlète comme un métier dans un but élitiste, en privilégiant le résultat et la performance. Il en tire sa subsistance moyennant une rémunération conformément à son statut professionnel. Son adhésion au club inscrit comme société à objet sportif se fait sur base d'un contrat professionnel tandis que son affiliation à la fédération peut être effectuée soit par l'entremise d'un club soit encore en sa qualité d'athlète indépendant.

De tout ce qui précède, l'applicabilité des rôles assignés à toutes les formes de pratiques sportives démontre que le sport est réel atout qui se situe au centre d'intérêt social.

\section{LE SPORT EN TANT QUE RECEPTACLE D'INTERET SOCIAL}

1. Le sport pris comme moyen du développement

Dans le cadre de l'intérêt social, l'apport du sport s'inscrit « dans le sport qui se décline sous toutes les formes à la fois spectacle, produit de consommation, moyen de publicité, loisir, et employeur. Incontestablement, une réalité sociale $»^{19}$. Considérant la production de richesse dans le football, on peut mentionner que « la patrimonialisation du football constitue un bouleversement du schéma classique du service public improductif vers un service public productif, support au développement national $»^{20}$.

Dans sa dimension internationale le sport ne caisse d'être considéré comme : un droit de l'homme qui s'est matérialisé dans la mise sur pied du Conseil consultatif de la FIFA sur le droits de l'homme en 2017, un moyen de lutte contre les différentes formes des discrimi-

18 Norbert ELIAS et Eric GUNNING, «La dynamique des groupes sportifs et l'exemple du football » in Sport et civilisation la violence maitrisée, éd. Fayard, France, 1998, p.294.

19 Franck NICOLLEAU, «Le pouvoir des fédérations sportives » thèse soutenue à l'université de Versailles Saint-Quentin-En-Yvelines des Sciences Juridiques et Politiques, du 2 juillet 2001, p.6.

20 Jean-Marie MUSONDA, « L'encadrement juridique de la pratique du sport et son impact sur le développement socio-économique en République Démocratique du Congo : cas du football »,Mémoire de Diplôme d'études approfondies en Droit/ UNILU/ Juillet 2018, p.18. 
nations ${ }^{21}$ et une activité au service du développement et de la paix. A cet effet, on peut encore noter dans le cadre du système des Nations-Unies du juillet 2002, le rapport impliquant « le sport au service du développement et de la paix : vers la réalisation des objectifs du millénaire pour le développement ayant abouti aux différentes recommandations aux Etats:

- Intégrer le sport et l'activité physique au programme de développement;

- Faire de la participation aux activités sportives un objectif et un instrument pour la réalisation des objectifs de développement pour le millénaire;

- Inclure des initiatives liées au sport dans les programmes des institutions des NationsUnies.

- Faciliter le développement de partenariats originaux mobilisant le sport au service du développement.

Pour Pascal Boniface, «Le sport est aujourd'hui un phénomène global par excellence dans la mesure où l'image d'un pays, sa popularité participent à sa puissance d'agir et sa globalisation désigne l'accroissement des échanges à travers la multiplication des compétitions internationales $»^{22}$.

\section{L'activité sportive comme activité économique}

Les enjeux économiques du sport nécessitent de prendre le football comme un modèle de vivier économique comme le souligne Jean-Remi Cognard : « activité sportive égale activité économique $»^{23}$. La question fondamentale s'intéresse sur l'imbrication du football à la protection économique en vue de pallier aux défis d'emploi et du développement social dans la ville de Lubumbashi dès lors l'on considère que « le sport représente un vivier d'emplois et de compétences, essentiels au développement économique du territoire $»^{24}$. Pour Jean-François Bourg et Jean-Jacques Gouguet, « le sport en général constitue désormais un phénomène social majeur, de même qu'une activité économique de première importance. Le football est devenu un produit dans la mesure où qu'il est le résultat de la logique de fonctionnement du mode de production capitaliste $»^{25}$. De ce qui précède, la logique financière qui découle de la formation, des transferts, de la billetterie et du merchan-

21 L'article 10 litera $(\mathrm{g})$ de la convention sur l'élimination de toutes les formes de discriminations à l'égard des femmes de 1971 offre les mêmes possibilités de participer activement aux sports et à l'éducation physique.

22 Pascal BONIFACE, « La géopolitique du sport » (in dictionnaire culturel du sport, sous dir. Michael Attali et Jean Saint-Martin) éd. Armand Colin, 2010, pp 307-311.

23 Jean-Remi COGNARD, op.cit., p.10.

24 Conseil économique social et environnement des Pays de Loire, Sport vecteur de lien social et rôle éducatif session du 21 au 22 juin 2012, p.15.

25 Jean-François BOURG et Jean-Jacques GOUGUET, « Economie » in dictionnaire culturel du sport, éd. Armand Colin, Paris, 2010, p.290. 
dising doit être au service de sécurisation économique et d'occupation de la jeunesse lushoise.

\section{Quelques actions du soft power}

Parmi les actions de soft power, c'est-à-dire qui ne recourent pas à la force légitime, l'exemple de l'Eglise catholique est fort actif dans l'encadrement des jeunes en rupture familiale. Nous avons fait allusion aux initiatives des prêtres salésiens de Lubumbashi et des frères xaveriens de la ville de Likasi qui gardent les jeunes dans leurs centres. Dans les trois Communes visitées nous avons : la maison Bakanja située dans la Commune de Lubumbashi, la maison des Jeunes située dans la Commune de la Rwashi et la Cité des jeunes située dans la Commune Kampemba. Au regard des actions entreprises pour l'encadrement des jeunes figurent : l'apprentissage de différents métiers (la soudure, la mécanique automobile et la maçonnerie), les enseignements des lectures et des calculs ainsi que plusieurs séances des activités de football qui jouent le rôle moteur dans la réalisation des perspectives de cohésion des muscles juvéniles. En plus, la Congrégation des prêtres crée des opportunités de contacts avec certaines familles pour la réinsertion familiale.

De ce qui précède, c'est essentiellement l'influence du sport et la création des centres d'accueil des jeunes en ruptures familiales qui sont des éléments d'intérêts indéniables qui entrent dans le cadre des mesures de sureté pour préserver l'ordre public en s'inscrivant dans la logique du professionnalisme de maintien de l'ordre public en marge des mécanismes coercitifs de l'Etats joliment appelé le hard power. Ce moyen conduit à inscrire la gestion de l'ordre public à l'aune des staffs dans une approche multidisciplinaire.

\section{PERSPECTIVES DE CONSOLIDATION DE LA SECURITE}

En dépit des rhétoriques sur l'implication du sport dans la lutte contre l'insécurité dans la ville de Lubumbashi, nous pouvons envisager avant de conclure que :

a) Le maintien de l'ordre public doit être une préoccupation de tous. Cet aspect doit faire objet de la sensibilisation de la population sur la prolifération du phénomène Staff qui est détourné de son sens approprié;

b) La coopération des habitants de Lubumbashi avec de nouvelles autorités doit être menée de manière pérennante et qu'à leurs tours les gouvernants doivent prendre des mesures préventives et des précautions sur les milieux qui abritent les staffs en vue de reléguer au livre d'histoire les interpellations des chefs des staffs ou leurs encadreurs qui s'articulent autour de procédures routinières;

c) L'occupation de la jeunesse par la création des centres sportifs en vue de valoriser les muscles non scolarisés en des muscles utiles au développement doit au centre de la vie nationale dès lors que l'aspect élitiste dans le domaine sportif ne cadre pas essentiellement avec les diplômes mais avec la performance physique et technique qui sont porteuses des enjeux économiques; 
d) La sensibilisation de la population sur la sacralité de la vie humaine convient de rester le seul moyen de valider la prise de conscience pouvant anticiper la création des staffs supplémentaires;

e) Le cantonnement des hommes en uniforme dans les casernes et l'applicabilité effective des permutations apporteront à notre entendement un vent nouveau dans la rupture de clientélisme dans le maintien de la sécurité et la tranquillité de la population luchoise en particulier et du peuple congolais en général.

\section{CONCLUSION}

Il est nécessaire de considérer la sécurité comme un bien public national qui se caractérise par la non-exclusion et la non-appropriation. Dans la mesure où elle renvoie à l'état d'esprit et est synonyme d'assurance, de calme, de confiance et de tranquillité, la sécurité converge à une situation qui ne présente aucun danger et qui respecte l'intégrité de l'individu. La prise en compte de cette approche est une dimension tant bénéfique pour le domaine du sport que pour l'ensemble de la ville de Lubumbashi. Ainsi, donc il y a lieu que toutes les actions convergent dans la gestion de l'ordre public. Le hard power et le soft power comme des pouvoirs qui doivent s'affirmer dans leur interaction en tant que des mécanismes complémentaires dans la lutte contre les mouvements sociaux négatifs. Pour ce faire le partenariat public et privé s'impose et doit représenter une opportunité moderne pour investir adéquatement leurs forces dissuasives rigides et souples au maintien de l'ordre public.

\section{REFERENCES BIBLIOGRAPHIQUES}

\section{TEXTES LEGAUX}

1. Constitution du 18 février 2006 modifiée par la loi n¹1/002 du 20 janvier 2011 portant révision de certains articles (in J.O. numéro spécial 52).

2. La convention unique sur les stupéfiants de 1961 telle modifiée par le protocole de 1972.

3. La convention sur les substances psychotropes ainsi que la convention des nations unies contre le trafic illicite de stupéfiants et de substances psychotropes de 1988.

4. La convention sur l'élimination de toutes les formes de discriminations à l'égard des femmes entrée en vigueur le 3 septembre 1981.

5. Loi n ${ }^{\circ} 11 / 023$ du 24 décembre 2011 portant principes fondamentaux relatifs et l'organisation et à la promotion des activités physiques et sportives en République Démocratique du Congo.

\section{OUVRAGES}

1. Gauthier de VILLERS, Jean-Claude WILLAME, Jean OMASOMBO et Erik KENNES, République Démocratique du Congo Chronique politique d'un entre- deux- guerres octobre 1996-juillet 1998, éd. L'Harmattan, Paris, 1998 
2. Jean-Remi COGNARD, Contrat de travail dans le sport professionnel, éd. Juris éditions, coll. Horssérie, Paris, 2012.

3. Niagalé GAGAYOKO-PENONE, Afrique: les stratégies française et américaine, L'Harmattan, Paris, 2003.

4. ONUDC, les conventions internationales relatives au contrôle des drogues, édition révisée 2013.

5. Paul MUSAFIRI, Pièges symboliques et violation des Droits Humaines en Droit positif congolais contribution critique à l'élaboration du Droit praxéologique, éd. La Dialectique, Lubumbashi, 2014.

6. Emmanuel TESSIER, Le stade en droit public recherche sur le régime juridique des enceintes sportives, éd. L'Harmattan, Paris, 2015.

\section{ARTICLES ET REVUES}

1. Alain FERRAND, « Mécénat » in dictionnaire culturel du sport, éd. Armand Colin, Paris, 2010.

2. Jean-François BOURG et Jean-Jacques GOUGUET, «Economie » in dictionnaire culturel du sport, éd. Armand Colin, Paris, 2010.

3. Jean-Marie DIKANGA KAZADI, « La gestion de l'ordre public dans la province du Katanga » in frontières et gouvernance sécuritaire pour le développement de la République Démocratique du Congo (dir Jean-Marie DIKANGA KAZADI et Emmanuel BANYWESIZE), éd. L'Harmattan, Paris, 2013.

4. Jean-Remi COGNARD, Contrat de travail dans le sport professionnel, Juris éditions, coll. Hors-série, 2012.

5. Joseph TSHIBASU PANDAMADI, « La problématique de la répression du trafic illicite des stupéfiants en RDC et son incidence dans l'ordre public national et international » in Revue Justitia Faculté de Droit/Université de Lubumbashi, 2018.

6. Norbert ELIAS et Eric GUNNING, "La dynamique des groupes sportifs et l'exemple du football» in Sport et civilisation la violence maitrisée, éd. Fayard, France, 1998.

7. Pascal BONIFACE, « La géopolitique du sport» in dictionnaire culturel du sport, (sous dir. Michael Attali et Jean Saint-Martin), éd. Armand Colin, 2010.

8. Thierry LARDINOIT et Gary TRIBOU, «Quelle est la spécificité de la gestion du sport? » in Revue française de Gestion dossier de gestion du sport, vol.30, n¹50, éd. Lavoisier, 2004.

\section{THESE ET MEMOIRE}

1. Franck NICOLLEAU, Le pouvoir des fédérations sportives, thèse soutenue à l'université de Versailles Saint-Quentin-En-Yvelines des Sciences Juridiques et Politiques, du 2 juillet 2001.

2. Jean-Marie MUSONDA, L'encadrement juridique de la pratique du sport et son impact sur le développement socio-économique en République Démocratique du Congo : cas du football, Mémoire de Diplôme d'études approfondies en Droit/ UNILU/ Juillet 2018. 